\title{
Managing Upper Respiratory Tract Infections
}

\section{Practice Without Evidence}

$I^{n}$

$n$ an era of growing emphasis on evidence-based medi-

cal practice, few practices are as "evidence-free" as the use of antibiotics for treating the common cold and related upper respiratory tract infections (URIs). Indeed, increasing attention has been focused on the strikingly high rate of antibiotic use for these conditions. ${ }^{1}$ The reasons we should be concerned include drug-related adverse effects and the economic burden associated with excess usage. The most troubling problem, however, is the role that improper use of antibiotics plays in facilitating the emergence of antimicrobial resistance.

One example is the recent proliferation of drug-resistant Streptococcus pneumoniae. Approximately $44 \%$ of clinical isolates of S. pneumoniae in the United States have either intermediate or high levels of penicillin resistance, ${ }^{2}$ and one of the most important risk factors for carriage of this resistant pathogen is prior antibiotic use.3,4

Curtailment of unnecessary antibiotic use thus is vitally important. Physicians prescribe antibiotics for more than $50 \%$ of their patients who have URIs or bronchitis, although these infections usually are caused by viruses, ${ }^{1}$ against which antibiotics have little or no clinical benefit. 5,6 In 1992 these two conditions accounted for 31\% of antibiotic prescriptions. ${ }^{7}$

If antibiotics are unnecessary in the vast majority of URI cases, why do physicians prescribe them? Many physicians are unclear about what role, if any, antibiotics should play in the management of URIs. ${ }^{8}$ Also, many patients expect to receive antibiotics, physicians worry about patients becoming dissatisfied, and physicians may not have enough time to discuss antibiotic use with their patients..$^{9,10}$

Could there be other reasons? To answer this question, in this issue Gonzales and colleagues report their efforts to identify characteristics of a patient's clinical presentation that influence the decision to prescribe antibiotics. ${ }^{11} \mathrm{~Pa}-$ tients from three ambulatory care practices were studied over 4 months. Patients with a history of chronic heart or lung disease, a coexistent antibiotic-responsive illness, or acute bronchitis were excluded. Clinicians prescribed antibiotics more often when the patient used tobacco, or reported green or yellow nasal discharge or green phlegm, or the clinician found a purulent nasal discharge or a tonsillar exudate. The more of these findings that were present, the more likely the clinician was to prescribe antibiotics.

The authors suggest that clinicians are influenced by these signs and symptoms because they believe the findings identify patients with URIs that are more responsive to antibiotics. Alternatively, patients could request antibiotics more frequently when they have these symptoms or learn about the clinician's findings. In fact, approximately $80 \%$ of patients believe antibiotics are effective for an infection in which there is discolored nasal discharge. ${ }^{12}$ Whether these findings influence clinician behavior or patient expectations or both, the bottom line is that evidence from well-done studies does not support the belief that these patients have antibiotic-responsive URIs. ${ }^{13,14}$

Perhaps clinicians in this study used purulent signs and symptoms as diagnostic clues for the presence of alternative, antibiotic-responsive conditions. Green phlegm or discolored nasal discharge are frequently seen in conditions such as pneumonia or sinusitis, and physicians may be influenced by these findings to prescribe antibiotics even if they do not specifically identify the condition as pneumonia or sinusitis. However, such an approach is not evidencebased. Purulent manifestations are weak predictors of these illnesses. ${ }^{15,16}$

How can we introduce evidence-based medicine into the outpatient management of colds and URIs? One way is through practice guidelines. The Centers for Disease Control and Prevention recently published guidelines for pediatric patients. ${ }^{17}$ Similar guidelines are urgently needed to help clarify when antibiotics are appropriate for adults. In this regard, it is interesting that more than $80 \%$ of patients in the study by Gonzales and colleagues were seen primarily by clinicians other than physicians. Though the authors report that physicians supervised all nonphysician visits and signed all prescriptions, it is likely that prescribing decisions often reflected the beliefs and practices of the nurses and nurse practitioners. This finding implies that efforts to change prescribing patterns need to be directed not only at physicians but also at other health care providers.

It is important to identify the beliefs that lead to unnecessary prescriptions for antibiotics, because changing these misconceptions may result in more appropriate use and a decrease in antimicrobial resistance. Although the data are limited, a study in Finland found that the proportion of group A streptococcal infections resistant to macrolide antibiotics decreased by nearly $50 \%$ following a national campaign to reduce macrolide use. ${ }^{18}$ This campaign, however, focused on only one class of antibiotics. More must be done to show that curtailing prescriptions in inappropriate settings and thus decreasing antibiotic use will be effective in decreasing antimicrobial resistance.

The unnecessary use of antimicrobial agents in the community continues to be of major concern. Although the factors that lead to inappropriate use are complex, attempts to identify and subsequently alter them must be pursued urgently. Only through the judicious use of antibiotics can we ensure their continued effectiveness in their future.Ebbing lautenbach, MD, MPH, Joshua P. Metlay, MD, PHD, Center for Clinical Epidemiology and Biostatistics, University of Pennsylvania Medical Center, Philadelphia, Penn.

\section{REFERENCES}

1. Gonzales R, Steiner JF, Sande MA. Antibiotic prescribing for adults with colds, upper respiratory tract infections, and bronchitis by ambulatory care physicians. JAMA. 1997;278:901-4. 
2. Doern GV, Brueggemann AB, Blocker M, et al. Clonal relationships among high-level penicillin-resistant Streptococcus pneumoniae in the United States. Clin Infect Dis. 1998;27:757-61.

3. Arason V, Kristinsson K, Sigurdsson J, Stefansdottir G, Molstad $\mathrm{S}$, Gudmundson S. Do antimicrobials increase the carriage rate of penicillin resistant pneumococci in children: cross sectional prevalence study. BMJ. 1996;313:387-91.

4. Arnold KE, Leggiadro RJ, Brieman RF, et al. Risk factors for carriage of drug-resistant Streptococcus pneumoniae among children in Memphis, Tennessee. J Pediatr. 1996;128:757-64.

5. Soyka LF, Robinson DS, Lanchant N, Monaco J. The misuse of antibiotics for treatment of upper respiratory tract infections in children. Pediatrics. 1975;55:552-6.

6. Verjeij TJM, Hermans J, Mulder JD. Effects of doxycycline in patients with acute cough and purulent sputum: a double-blind, placebo-controlled trial. Br J Fam Pract. 1994;44:400-4.

7. McCaig LF, Hughes JM. Trends in antimicrobial drug prescribing among office-based physicians in the United States. JAMA. 1995; 273:214-9.

8. Mainous AG, Hueston WJ, Eberlein C. Colour of respiratory discharge and antibiotic use. Lancet. 1997;350:1077.

9. Hamm RM, Hicks RJ, Bemben DA. Antibiotics and respiratory infections: are patients more satisfied when expectations are met? J Fam Pract. 1996;43:56-62.

10. Palmer DA, Bauchner HR. Parents' and physicians' views on antibiotics. Pediatrics. 1997;99:E6.
11. Gonzales R, Barrett PH, Steiner JF. The relation between purulent manifestations and antibiotic treatment of upper respiratory tract infections. J Gen Intern Med. 1999;14:151-6.

12. Mainous AG, Zoorob RJ, Oler MJ, Haynes DM. Patient knowledge of upper respiratory infections: implications for antibiotic expectations and unnecessary utilitzation. J Fam Pract. 1997;45: 75-83.

13. Todd JK, Todd N, Damato J, Todd WA. Bacteriology and treatment of purulent nasopharyngitis: a double-blind placebo-controlled evaluation. Pediatr Infect Dis. 1984;3:226-32.

14. Steinwegg KK. Natural history and prognostic significance of purulent rhinitis. J Fam Pract. 1983;17:61-4.

15. Metlay JP, Kapoor WN, Fine MJ. Does this patient have community-acquired pneumonia? Diagnosing pneumonia by history and physical examination. JAMA. 1997;278:1440-5.

16. Williams JW, Simel DL. Does this patient have sinusitis? Diagnosing acute sinusitis by history and physical examination. JAMA. 1993;270:1242-6.

17. Dowell SF, Marcy SM, Phillips WR, Gerber MA, Schwartz B. Principles of judicious use of antimicrobial agents for pediatric upper respiratory tract infections. Pediatrics. 1998;101:163-5.

18. Seppala H, Klaukka T, Vuopio-Varkila I, et al. The effect of changes in the consumption of macrolide antibiotics on erythromycin resistance in group A streptococci in Finland. N Engl J Med. 1998;337: 441-6. 